\title{
Microstimulation at the bone-implant interface upregulates osteoclast activation pathways
}

\author{
Vincent A. Stadelmann, Alexandre Terrier, Dominique P. Pioletti* \\ Laboratory of Biomechanical Orthopedics EPFL-HOSR, Institute of Translational Biomechanics, Station 15, \\ Ecole Polytechnique Fédérale de Lausanne, 1015 Lausanne, Switzerland
}

Received 21 August 2007; revised 20 September 2007; accepted 22 September 2007

Available online 5 October 2007

\begin{abstract}
Peri-implant bone resorption after total joint arthroplasty is a key parameter in aseptic loosening. Implant wear debris and biomechanical aspects have both been demonstrated to be part of the bone resorption process. However, neither of these two parameters has been clearly identified as the primary initiator of peri-implant bone resorption. For the biomechanical parameters, micromotions were measured at the boneimplant interface during normal gait cycles. The amplitude of the micromotions was shown to trigger differentiation of bone tissues. So far no data exists directly quantifying the effect of micromotion and compression on human bone. We hypothesize that micromotion and compression at the bone-implant interface may induce direct activation of bone resorption around the implant through osteoblasts-osteoclasts cell signaling in human bone. This hypothesis was tested with an ex vivo loading system developed to stimulate trabecular bone cores and mimic the micromotions arising at the bone-implant interface. Gene expression of RANKL, OPG, TGFB2, IFNG and CSF-1 was analyzed after no mechanical stimulation (control), exposure to compression or exposure to micromotions. We observed an 8-fold upregulation of RANKL after exposure to micromotions, and downregulation of OPG, IFNG and TGFB2. The RANKL:OPG ratio was upregulated 24-fold after micromotions. This suggests that the micromotions arising at the bone-implant interface during normal gait cycles induce a bone resorption response after only $1 \mathrm{~h}$, which occurs before any wear debris particles enter the system.
\end{abstract}

(C) 2007 Elsevier Inc. All rights reserved.

Keywords: Bone resorption; Orthopedic implant; Mechanical stimulation; Gene expression; Micromotions

\section{Introduction}

After total joint arthroplasty, a radiolucent zone is frequently observed at the interface between bone and implant [4,29]. This radiolucent zone is associated with a progressive peri-implant bone resorption. The implant fixation is affected, therefore inducing a risk of aseptic loosening. This becomes a serious problem as aseptic loosening accounts for more than two-third of hip revisions in Sweden, a country where an extensive implant register has been set up for many years [16].

Two hypotheses are generally used to explain peri-implant bone resorption. The first hypothesis focuses on a biological reaction to wear particles. Numerous studies have shown that the debris after implant wear induces inflammatory reactions in

\footnotetext{
* Corresponding author. Fax: +41 216938660.

E-mail address: dominique.pioletti@epfl.ch (D.P. Pioletti).
}

the tissues surrounding the implant [2,7]. Bone formation may also be impaired by the presence of particles, such as titanium debris which were shown to induce apoptosis to osteoblasts culture in vitro [24]. In all cases, particulate debris accumulate in the tissue surrounding the implant. Upon accumulation, a chain of cellular events is triggered within the tissue leading to periprosthetic osteolysis and implant loosening [15]. The second hypothesis used to explain peri-implant bone resorption is based on biomechanical considerations. A stiff metallic implant in a load bearing bone considerably changes the mechanical state of the bone. Based on a numerical approach, Huiskes and Nunamaker showed that bone resorption around the implant is associated with high peak stresses immediately postoperatively [8]. The bone structure is affected by the new stress patterns around an implant. Numerical models predict bone loss around the implant based only on these modified mechanical patterns [33]. 
Mechanical effects play certainly also a crucial role at the bone-implant interface. The pumping action of the implant during gait cycle causes load fluctuation within the hip joint fluid. Using a numerical model, the interfacial compressive stress involved was found to be between $2 \times 10^{-3}$ and $0.1 \mathrm{MPa}$ [27]. Beside compressive stress, micromotions at the interface have been suspected to play a key role in tissue differentiation around the implant. It has been calculated that micromotions between 5 and $100 \mu \mathrm{m}$ occur at the bone-hip implant interface during normal gait cycles [27]. Mandell et al. analyzed the case of conical-collared intramedullary hip stem and reported micromotions up to $163 \mu \mathrm{m}$ in the worst design [17]. From an experimental point of view, Baleani et al. quantified bone- implant micromotions under torsional load with position transducers in a hip implant model. A maximum of $56 \mu \mathrm{m}$ was measured in uncemented stems [1]. Finally, with in vivo models, Jasty et al. found that micromotions lower than $40 \mu \mathrm{m}$ favor bone formation in dog, while micromotions higher than $100 \mu \mathrm{m}$ lead to the creation of a fibrous tissue [10].

Based on clinical observations, numerical and experimental biomechanical analysis and in vivo experiments, there is strong evidence to support the theory that micromotions and compressive stress at the bone-implant interface play an important role in the process of peri-implant bone resorption. However, so far no data exist quantifying directly the effect of micromotion and compression on human bone. Therefore we
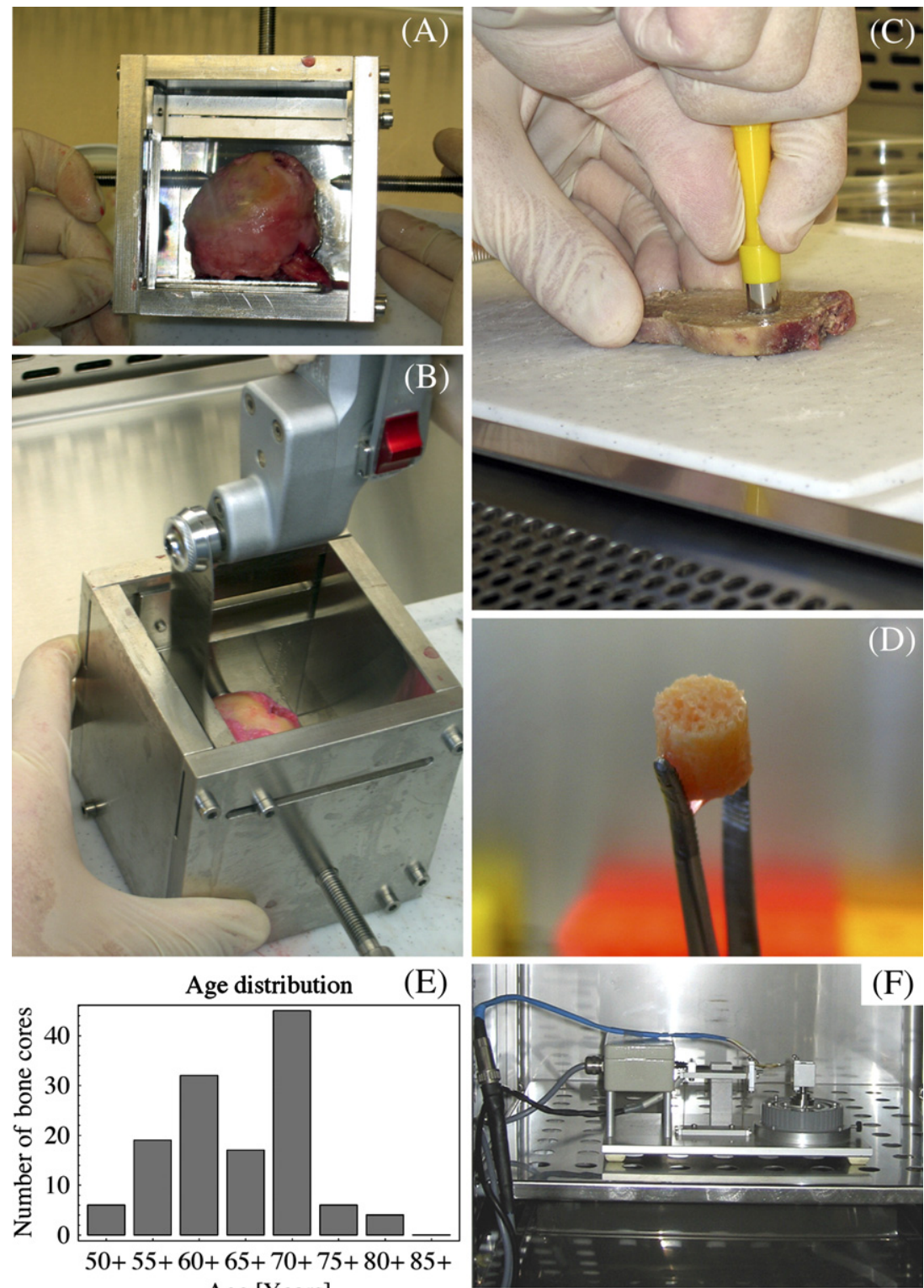

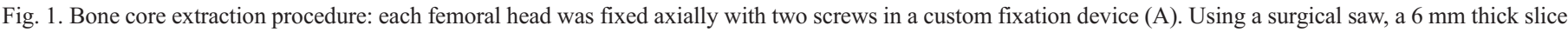

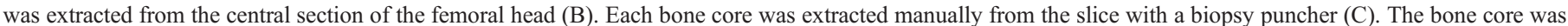

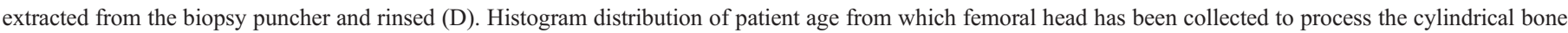

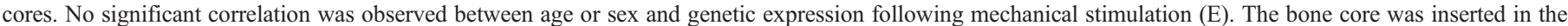

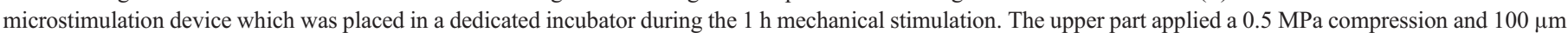
micromotions on the top of the bone core. The device was controlled with an external computer $(\mathrm{F})$. 
hypothesize that micromotion and compression at the boneimplant interface may induce direct activation of bone resorption around the implant through osteoblasts-osteoclasts cells signaling in human bone. This hypothesis was tested with an ex vivo loading system using human bone samples.

\section{Materials and methods}

\section{Bone samples preparation}

Twenty-five human femoral heads were obtained from the Hôpital Orthopédique de la Suisse Romande following total hip prosthesis procedures (Ethical Protocol 51/01, University of Lausanne). In the next $4 \mathrm{~h}$ following the sample collection, each femoral head was fixed axially in a custom fixation device and a central section of $6 \mathrm{~mm}$ thick slice was extracted with a surgical saw. Then, 4 to 16 trabecular bone cores of $3 \mathrm{~mm}$ radius and $6 \mathrm{~mm}$ height were extracted from the slice with a biopsy puncher (Shoney Scientific, Pondicherry, India) at $15 \mathrm{~mm}$ from the cortical bone layer (see Figs. 1A-D). The bone cores were then incubated overnight in DMEM (Sigma, Buchs, Switzerland) containing $10 \%$ of fetal bovine serum (Sigma) and $1 \%$ of PSF (100×, $10,000 \mathrm{U} / \mathrm{ml}$ penicillin, 10,000 $\mu \mathrm{g} / \mathrm{ml}$ Strepzin, $25 \mu \mathrm{g} / \mathrm{ml}$ Fungizone) (GibcoBRL, New York, USA) at $37{ }^{\circ} \mathrm{C}, 5 \% \mathrm{CO}_{2}, 90 \% \mathrm{H}_{2} \mathrm{O}$.

\section{Initial gene expression level}

Nine samples were used to control the initial gene expression level after sample preparation. Three samples were collected immediately after punching, three samples $24 \mathrm{~h}$ after punching and three samples $48 \mathrm{~h}$ after punching. These nine samples were not mechanically stimulated but placed in $1 \mathrm{ml}$ Trizol (Invitrogen $\mathrm{AG}$, Switzerland) and stored at $-80{ }^{\circ} \mathrm{C}$ for later ribonucleic acid (RNA) extraction.

\section{Bone samples stimulation}

A device was developed to apply combined compression and micromotions mode on the surface of trabecular bone samples simulating then the mechanical situation arising at the bone-implant interface [23]. Briefly, the device consists of a bottom fixed and top moving plates with bone core placed in between (Fig. 1F). A $0.5 \mathrm{MPa}$ compression was applied from top and sinusoidal micromotion of $100 \mu \mathrm{m}$ at a frequency of $1 \mathrm{~Hz}$ were applied on the top bone surface. As the mechanical stimulation targeted micromotion and compression, a theoretical evaluation of the loading modes was performed. A Finite Element Analysis was then developed to evaluate the mechanical stimulation in the bone core corresponding to the experimental boundary conditions. Because of the relative low frequency and high porosity, the inertial and fluid effects were omitted in this numerical study. The bone sample was assumed elastic, homogeneous and isotropic, and the contact was assumed to follow a Coulomb friction law. The elastic modulus was $250 \mathrm{MPa}$ [19] and the bone-metal friction coefficient was $0.2[14,28]$. For the compression case, the volume average of the octahedral normal and shear strains was respectively 286 and 1190 microstrains. When micromotions were added to compression, normal strains were not affected, but shear strains increased by $7 \%$ (Fig. 2).

The bone cores were separated randomly into three groups: control, compression and micromotion. Bone cores from the control group were incubated for $1 \mathrm{~h}$ at rest in $1 \mathrm{ml}$ culture medium (control). Bone cores from the compression group were incubated in $1 \mathrm{ml}$ culture medium and a compression of $0.5 \mathrm{MPa}$ was applied vertically on the sample in a special surgical steel chamber during $1 \mathrm{~h}$ (compression). Bone cores from the micromotion group were incubated in $1 \mathrm{ml}$ culture medium. A $0.5 \mathrm{MPa}$ compression was applied from top and sinusoidal micromotion of $100 \mu \mathrm{m}$ at a frequency of $1 \mathrm{~Hz}$ were applied on the top surface during $1 \mathrm{~h}$ (micromotion). The parameters of the sample stimulations were chosen according to the results of previous numerical studies performed in our laboratory [27] and corresponded to a normal load during gait cycles.

\section{RNA extraction}

Immediately after the stimulations, each sample was placed in individual tube containing $1 \mathrm{ml}$ Trizol and stored at $-80^{\circ} \mathrm{C}$. The bone samples were mixed with a stainless steel bead (QIAGEN GmbH, Germany) deposited in each tube by shaking the tubes three times in the MM300 Mixer Mill (Retsch GmbH and Co, Hann, Germany) at $30 \mathrm{~Hz}$ during $30 \mathrm{~s}$. Then, $0.2 \mathrm{ml}$ of chloroform (SigmaAldrich, Switzerland) was added to the homogenate. The tubes were shaked manually, and after phase separation, $0.5 \mathrm{ml}$ of the upper phase was transferred in new tubes where $0.5 \mathrm{ml}$ of ice-cold isopropanol (Sigma-Aldrich) was added. Tubes were vortexed and stored overnight at $-80{ }^{\circ} \mathrm{C}$. RNA was isolated and purified with NucleoSpin columns (Macherey-Nagel, Düren, Germany) according to the protocol furnished by the manufacturer. The concentration and quality of the extracted RNA were measured with a biophotometer (Eppendorf AG, Germany). RNA integrity was verified by optical density (OD) absorption ratio A260/280 $\mathrm{nm}$. OD between 1.7 and 2.2 was chosen as a quality criteria for inclusion (adapted from Cambridge Systems Biology Centre, Cambridge, UK). RNA was eluted in $40 \mu \mathrm{l}$ of RNase-free water and stored at $-80^{\circ} \mathrm{C}$ until further processing.

\section{First strand synthesis}

For each sample, $5 \mu 1$ of total RNA was reverse-transcribed using the Taqman Universal polymerase chain reaction (PCR) reagents with random hexamers (Applied Biosystems, Foster City, CA). Reaction volumes were fixed

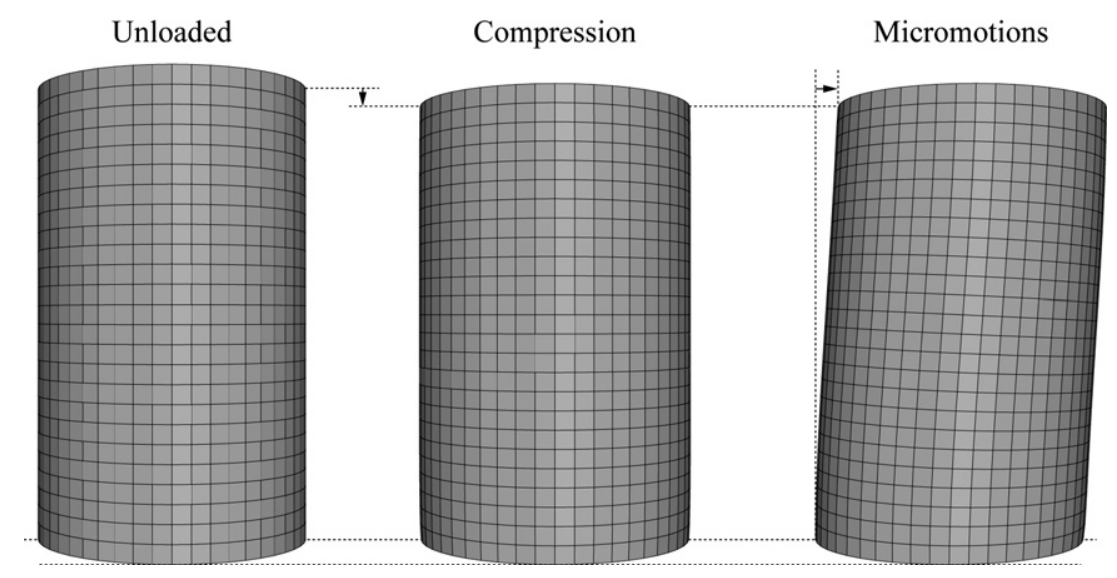

Fig. 2. A Finite Element Analysis provided an estimation of the octahedral normal and shear strains, for the compression and micromotions cases. When micromotions were added to compression, normal strains did not changed, but shear strains increased by $7 \%$. For illustration purpose, the deformation of the finite element mesh is magnified by 20 . 
at $50 \mu 1$ according to the protocol provided by the supplier. The thermal cycler PCT-0100 (MJ Research, Waltham, MA) was programmed as follows: $25^{\circ} \mathrm{C}$ $10 \mathrm{~min}, 48^{\circ} \mathrm{C} 30 \mathrm{~min}$, and $95^{\circ} \mathrm{C} 5 \mathrm{~min}$.

\section{Quantitative polymerase chain reaction ( $q P C R$ )}

The following gene expressions were quantified: RANK ligand (RANKL), osteoprotegerin (OPG), interferon-gamma (IFNG), colony stimulating factor 1 (CSF-1) and transforming growth factor beta2 (TGFB2) as target genes and glyceraldehyde-3-phosphate dehydrogenase (GAPDH) as non-regulated reference gene (housekeeping gene).

Specific primers for each gene were designed with the Primer Express ${ }^{\circledR}$ software (Applied Biosystems) and purchased from Integrated DNA Technologies (Coralville, IA). An additional sequence of $18 \mathrm{bp}$ was added to the $5^{\prime}$ end of every forward primer to use the Amplifluor Universal Detection System (Intergen Discovery Products, Purchase, NY). PCR reactions were performed in $25 \mu 1: 5 \mu 1$ of first strand, $12.5 \mu 1$ of TaqMan Universal PCR Master Mix (Applied Biosystems), and $7.5 \mu 1$ of the primers working solution. Thermal cycle conditions were $50{ }^{\circ} \mathrm{C} 2 \mathrm{~min}, 95^{\circ} \mathrm{C} 10 \mathrm{~min}$, then 50 cycles at $95{ }^{\circ} \mathrm{C} 15 \mathrm{~s}$, $60{ }^{\circ} \mathrm{C} 1 \mathrm{~min}$. Amplifications were monitored with the ABI Prism 7700 (Applied Biosystems). Measurements were performed in duplicates.

\section{Gene expression analysis and statistics}

The threshold cycle $C_{\mathrm{T}}$ was measured for each gene and each sample from the PCR amplification curve with a standard routine. $C_{\mathrm{T}}$ table was analyzed for each gene in each experiment: if duplicates from one cDNA presented a difference larger than 3 cycles (due to experimental artifacts such as limited pipetting precision, presence of bubble in the well, etc.), the measure was dismissed; otherwise $C_{\mathrm{T}}$ was calculated as the duplicate mean. If $C_{\mathrm{T}}$ was greater than 35 cycles, the measure was dismissed.

Relative gene expressions were calculated with the $2^{-\Delta \Delta \mathrm{CT}}$ method [22] with GAPDH as housekeeping gene. As the level of GAPDH expression may be influenced by the mechanical stimulation and consequently would render invalid its use as reference gene, in a pilot study we normalized the GAPDH expression by the level of $18 \mathrm{~S}$ expression. No effect of mechanical stimulation was observed on GAPDH expression (data not shown). Unless $18 \mathrm{~S}$ is similarly regulated as GAPDH through mechanical stimulation, which seems to be unlikely, GAPDH gene expression can be used as a stable reference gene in this experiment. We used a randomization of the differences and one-way ANOVA to compare the gene expressions of the different groups [6]. All values were then normalized to the expression of the control group. All mathematical operations and statistical analysis were performed using Mathematica ${ }^{\circledR}$ (Wolfram Research, Inc. USA). A $p$-value lower than 0.05 was considered significant while $p$-value lower than 0.1 was considered as a strong trend.

\section{Results}

In the following results, we report normalized gene expressions as mean \pm standard error of the mean (SEM).

\section{Sample collection}

The bone core samples were obtained from femoral heads of male and female patients between 50 and 80 years old (Fig. 1E). No significant correlation was observed between age or sex and genetic expression. Table 1 summarizes the number of cylindrical cores in each group. One hundred and

Table 1

Number of bone cores used for each condition

\begin{tabular}{llll}
\hline \# of bone cores & Control & Compression & Micromotion \\
\hline 112 & 62 & 20 & 30 \\
\hline
\end{tabular}

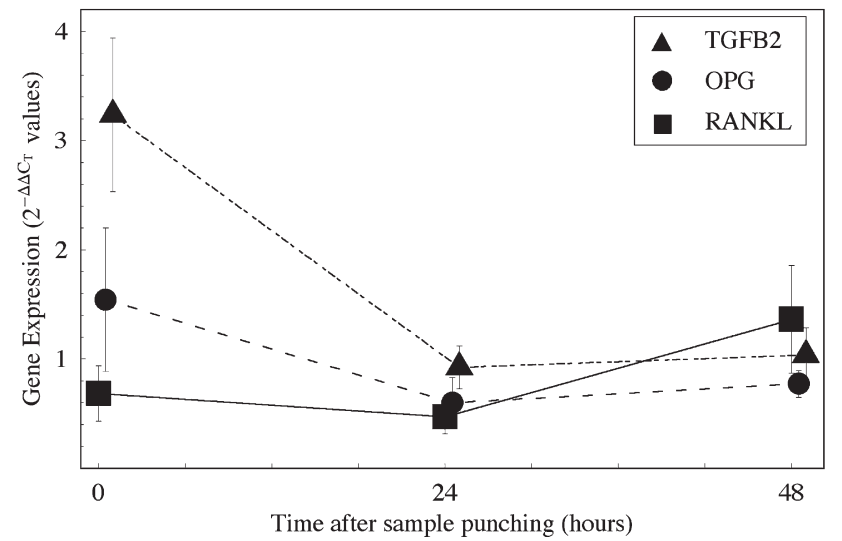

Fig. 3. Relative gene expression of TGFB2, OPG and RANKL quantified by RTPCR after 0,24 and $48 \mathrm{~h}$ of incubation. The results are shown as $2^{-\Delta \Delta C T}$ values and plotted as mean \pm SEM of each individual experiment.

twelve samples were used in the main experiment. Fifty-four samples passed the quality criteria (A260/A280) and were used for normalized gene expression analysis comparison between control, compression and micromotion regimen.

\section{Initial gene expression level}

As the process of bone core extraction may affect the gene expression of the sample, nine samples were used to evaluate the initial and $24 \mathrm{~h}$ gene expressions level after samples preparation and compare it to the value obtained $48 \mathrm{~h}$ after sample preparation. Immediately after sample punching, gene expressions were the following: RANKL $0.68 \pm 0.25$, OPG $1.5 \pm 0.7$, TGFß $3.2 \pm 0.7$ compared to the $48 \mathrm{~h}$ values. After $24 \mathrm{~h}$, the expressions were: RANKL $0.5 \pm 0.2$, OPG $0.6 \pm 0.2$, TGF $\beta$ $0.9 \pm 0.2$ compared to the $48 \mathrm{~h}$ values. Finally, after $48 \mathrm{~h}$, when we compare the values between each sample, the expressions became: RANKL $1.4 \pm 0.5$, OPG $0.77 \pm 0.12$, TGF $\beta 1.0 \pm 0.2$ (Fig. 3). None of the expressions at $48 \mathrm{~h}$ was significantly different from one, meaning that the gene expression of all core samples was similar. None of the expression levels at $24 \mathrm{~h}$ was significantly different from those at $48 \mathrm{~h}$. The variance of the expression levels was significantly different than those immediately after punching. These results suggest that, after 1 or 2 days of incubation, the pool of bone samples is in a homogenous state of genetic expression at least for the tested genes. All the samples were then incubated $24 \mathrm{~h}$ after extraction prior to the $1 \mathrm{~h}$ mechanical test.

\section{Gene expression according to the mechanical stimulus}

The relative gene expression for the selected genes and mechanical stimulations are presented in Fig. 4. RANKL expression was upregulated $2.8 \pm 0.9$-fold in the compression group and upregulated $8 \pm 2.8$-fold in the micromotion group when compared to control. The differences between compression and control, and between micromotion and control were significant, whereas there was a strong trend suggesting that the expression level between micromotion and compression was different. 

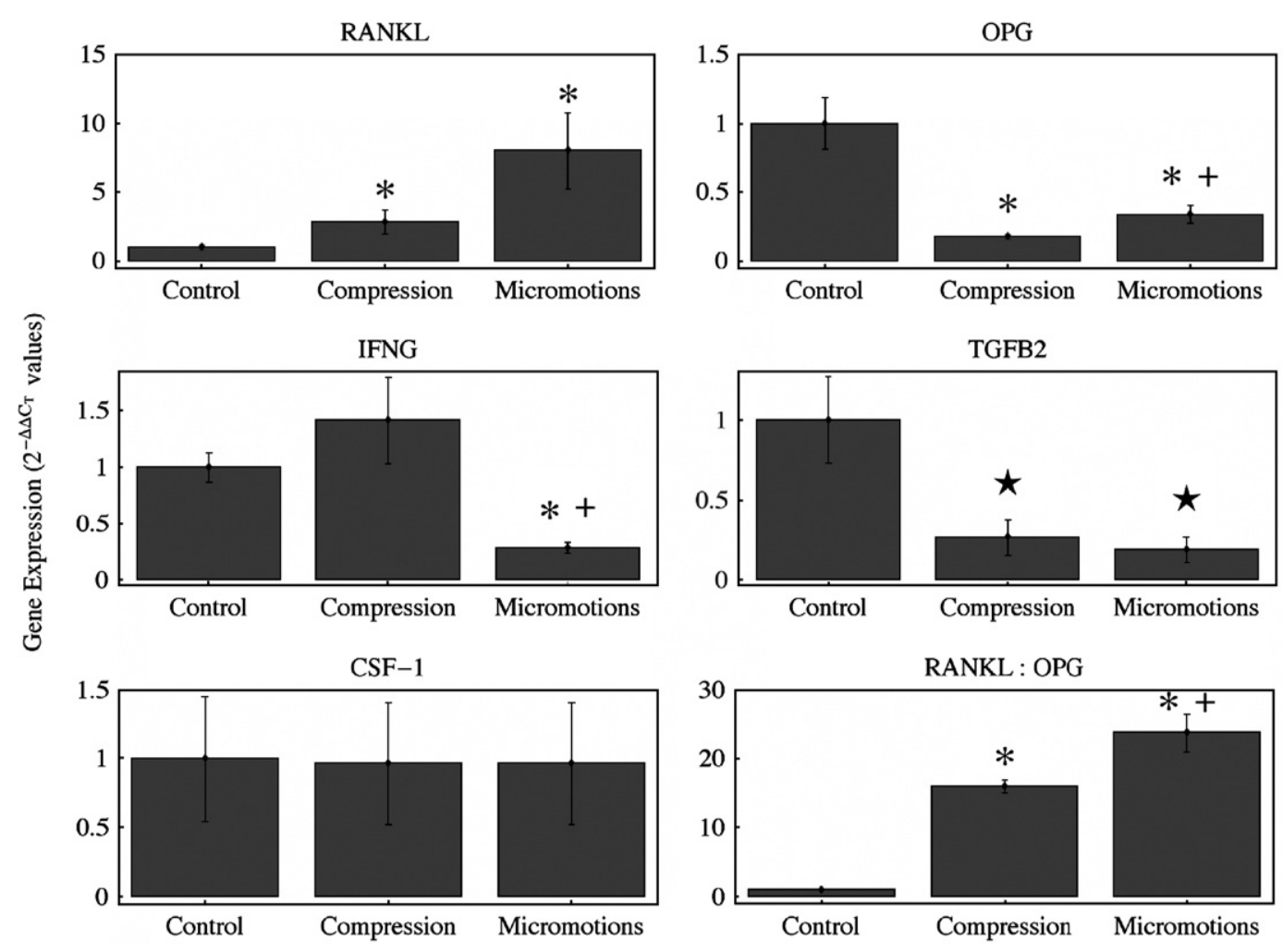

Fig. 4. Relative gene expression of RANKL, OPG, IFNG, TGFB2 and RANKL:OPG expression ratio quantified by RT-PCR after $1 \mathrm{~h}$ of incubation (control), $1 \mathrm{~h}$ of $0.5 \mathrm{MPa}$ compression or $1 \mathrm{~h}$ of $0.5 \mathrm{MPa}$ compression $+100 \mu \mathrm{m}$ micromotion (micromotion). The results are shown as $2^{-\Delta \Delta \mathrm{CT}}$ values and plotted as mean $\pm \mathrm{SEM}$ of each individual experiment. Symbols: ${ }^{*}(p<0.05$ vs. control), $+(p<0.05$ vs. compression), $\star$ (trend at $p<0.1$ level vs. control), $\dagger$ (trend at $p<0.1$ level vs. compression).

OPG expression was dramatically downregulated $0.18 \pm$ 0.02 -fold in the compression group and downregulated $0.34 \pm$ 0.07 -fold in the micromotion group when compared to control. The differences between compression and control, micromotion and control, and micromotion and compression were significant.

TGFB2 expression was downregulated $0.3 \pm 0.1$-fold in the compression group and downregulated $0.2 \pm 0.08$-fold in the micromotion group when compared to control. The difference between compression and control and micromotion and control was not significant but the $p$-value $(p=0.08)$ suggested a strong trend towards significant differences.

IFNG expression was not significantly changed in the compression group $(1.4 \pm 0.4)$ and was downregulated $0.29 \pm$ 0.05 -fold in the micromotion group when compared to control. The differences between micromotion and control and micromotion and compression were significant.

CSF-1 was not significantly affected in the compression group $(1 \pm 0.5)$ nor in the micromotion group $(1 \pm 0.5)$ when compared to the control group.

\section{Discussion}

A clinical study showed that up to $14 \%$ bone loss arose during the first 3 months after total hip arthroplasties [34]. In parallel, rapid early migrations have been detected by roentgen stereophotogrammetry in many asymptotic hips, often as early as 4 months postoperatively $[11,18]$. The migrations have been found to predict an increased risk of clinical loosening. The fate of an orthopedic implant seems then to be determined at an early stage, probably before any wear particles are produced, and is certainly related to mechanical factors. Micromotion at the bone-implant interface could then be considered as good candidates to explain peri-implant bone resorption and was indeed identified as key players in animal studies.

However, no information has so far confirmed these results for human bone as the only information available is based on tissue retrieval obtained from failed implants, the last stage of the degeneration process [31]. Based on a original ex vivo approach, the present study evaluates the micromotion effect on bone resorption for human samples and verifies if resorption could be initiated immediately after surgery, when no wear particles are part of the process.

We hypothesized that micromotions could induce an upregulation of genes involved in osteoclastic bone resorption. Therefore, we analyzed the expression ratios of the RANKL/OPG signaling system, as well as the expression ratios of TGFB2, IFNG and CSF-1. We used a control group, a compression group and a micromotion group, mimicking the situation arising around femoral stems of hip implants during gait cycles [27].

RANKL is a critical factor for late stage osteoclasts differentiation and activation. RANKL was shown to be expressed by osteoblasts after mechanical or hormonal stimulations [26]. OPG, the decoy receptor of RANKL produced by osteoblasts, is 
a powerful inhibitor of osteoclasts formation in vivo and in vitro [30]. Our results show that micromotion and compression dramatically increase RANKL expression suggesting that the number and the activity of osteoclasts at the surrounding implant is increased by micromotion and compression in normal gait conditions. We also observed a downregulation of OPG expression after exposition to compression alone or to micromotion suggesting that the balancing effect of RANKL by OPG is decreased.

Experimental and mathematical evidences have recently shown that OPG and RANKL expressions are regulated inversely in osteoblasts and that bone remodeling rate is probably determined by the RANKL:OPG ratio [3,12,13]. Our results are in concordance with these observations: RANKL and OPG are expressed inversely after exposition to compression alone or after compression and micromotion, as discussed previously. We observed that the RANKL:OPG ratio is significantly increased ten-fold by compression and significantly more than twenty-fold by compression and micromotion, suggesting that micromotions are potent activators of high bone turnover rate. Our observations on the regulation of RANKL/OPG by compression and micromotions suggest that the number of osteoclasts is enhanced, and bone turnover rate is increased in the periprosthetic area with normal gait cycle conditions. This might be one of the causes of the observed bone resorption around orthopedic implants.

Osteoclasts and osteoblasts activities are strongly correlated in vivo, and an increased osteoclastic activity might induce an osteoblastic response. Similarly, micromotion may affect osteoblastic recruitment. Therefore we also analyzed the expression of bone formation signaling molecules such as TGFB2 and IFNG. TGFB2 is expressed by osteoblasts and has contrasting effects on osteoblasts and osteoclasts. TGFB2 is a bone formation promoting molecule operating through chemotactic attraction of osteoblasts and enhancement of osteoblasts proliferation at the early stages of differentiation [9]. In cell culture, IFNG was shown to inhibit osteoblastic cell function [5] and potently inhibit osteoclasts formation. In bone explants, it inhibits osteoclasts differentiation [32]. We observed a downregulation of TGFB2 after exposure to compression alone and after compression plus micromotion, suggesting that micromotion at the implant interface does not increase recruitment of osteoblasts through TGFB2 signaling. TGFB2 was shown to be a promoter of osteoclasts formation by increasing the sensitivity of progenitors to RANKL (through upregulation of RANK in preosteoclastic cells) $[25,35]$. TGFB2 can also inhibit osteoclasts formation through a downregulation of the RANKL:OPG ratio [35]. In our analysis, RANKL:OPG is increased while TGF $\beta$ is decreased which is concordant. Our measures show that compression and micromotion decrease IFNG expression by two thirds. The consequence is then difficult to interpret in the peri-implant situation as the effect could be either a decrease of bone formation through inhibition of osteoblastic function or a decrease of bone resorption through inhibition of osteoclastic differentiation. Further studies are needed to clarify the role of this molecule.

The aim of the experimental setup used here was to simulate the mechanical situation at bone-implant interfaces using $e x$ vivo bone samples. The amplitudes of the applied compression and micromotion were set to measured or calculated values. The main drawback of the experimental set-up was the unknown consequence of preparation procedures and ex vivo incubation on bone cell function. We however showed that at least there is an initial homogeneous level of gene expression between samples. We can then assume that we have a consistency of initial conditions in our experiment. However, certainly due to the inherent biological variability, we obtained variable quality of extracted RNA, which may affect the gene expression quantification. It has to be mentioned that quantification of gene expression is only one part of the biological reaction to mechanical stimulus due to possible different post-transcriptional events. However, to our knowledge, no other experimental design allows one to study the effect of micromotion on the bone-implant interface with living human samples. These challenging technical difficulties were solved by a posteriori controls of RNA quality and variability of gene expression duplicates. More than $50 \%$ of the samples were discarded during these control procedures. It implied that paired-control statistical designs could not be use and that a large number of samples had to be processed to overcome the inter-specimen variations and to observe significant differences in the gene expression.

To conclude, our results suggest that micromotion at the bone-implant interface during normal gait cycles induce a rapid bone resorption response after only $1 \mathrm{~h}$, which occurs before any wear debris particles could enter the system. These results confirm our initial hypothesis.

Based on these results, we proposed that blocking locally the osteoclastic resorption through the action of bisphosphonate may prevent the peri-implant osteolysis. The first results of our animal model of implants used as bisphosphonate delivery systems tend to confirm this idea $[20,21]$.

\section{Acknowledgments}

Project no. 04-P2 was supported by the AO Research Fund of the AO Foundation, Davos, Switzerland. We thank MarcOlivier Montjovent and Sandra Jaccoud for technical assistance and Tyler Thacher for English editing.

\section{References}

[1] Baleani M, Cristofolini L, Toni A. Initial stability of a new hybrid fixation hip stem: experimental measurement of implant-bone micromotion under torsional load in comparison with cemented and cementless stems. J Biomed Mater Res 2000;50:605-15.

[2] Clarke IC, Campbell P, Kossovsky N. Debris-mediated osteolysis-a cascade phenomenon involving motion, wear, particulates, macrophage induction, and bone lysis. In: St. John KR, editor. Particulate debris from medical implants: mechanisms of formation and biological consequences, ASTM STP 1144. Philadelphia: American Society for Testing and Materials; 1992. p. 7-26.

[3] Fazzalari NL, Kuliwaba JS, Atkins GJ, Forwood MR, Findlay DM. The ratio of messenger RNA levels of receptor activator of nuclear factor kappaB ligand to osteoprotegerin correlates with bone remodeling indices in normal human cancellous bone but not in osteoarthritis. J Bone Miner Res 2001;16:1015-27. 
[4] Freeman MAR, Bradley GW, Revell PA. Observations upon the interface between bone and polymethylmethacrylate cement. J Bone Joint Surg Br 1982;64:489-93.

[5] Hirose K, Hanazawa S, Amano S, Kikuchi H, Takeshita A, Murakami Y, et al. Recombinant interferon-gamma is a potent inhibitor of osteoblastic cell functions. Meikai Daigaku Shigaku Zasshi 1989;18:296-301.

[6] Hooton JW. Randomization tests: statistics for experimenters. Comput Methods Programs Biomed 1991;35:43-51.

[7] Horikoshi M, Macaulay W, Booth RE, Crossett LS, Rubash HE. Comparison of interface membranes obtained from failed cemented and cementless hip and knee prostheses. Clin Orthop Relat Res 1994:69-87.

[8] Huiskes R, Nunamaker D. Local stresses and bone adaption around orthopedic implants. Calcif Tissue Int 1984;36(Suppl 1):S110-7.

[9] Janssens K, ten Dijke P, Janssens S, Van Hul W. Transforming growth factor-betal to the bone. Endocr Rev 2005;26:743-74.

[10] Jasty M, Bragdon C, Burke D, OConnor D, Lowenstein J, Harris WH. In vivo skeletal responses to porous-surfaced implants subjected to small induced motions. J Bone Joint Surg Am 1997;79A:707-14.

[11] Karrholm J, Borssen B, Lowenhielm G, Snorrason F. Does early micromotion of femoral stem prostheses matter? 4-7-year stereoradiographic follow-up of 84 cemented prostheses. J Bone Joint Surg Br 1994;76: 912-7.

[12] Komarova SV. Mathematical model of paracrine interactions between osteoclasts and osteoblasts predicts anabolic action of parathyroid hormone on bone. Endocrinology 2005;146:3589-95.

[13] Komarova SV, Smith RJ, Dixon SJ, Sims SM, Wahl LM. Mathematical model predicts a critical role for osteoclast autocrine regulation in the control of bone remodeling. Bone 2003;33:206-15.

[14] Kuiper JH, Huiskes R. Friction and stem stiffness affect dynamic interface motion in total hip replacement. J Orthop Res 1996;14:36-43.

[15] Lerouge S, Huk O, Yahia L, Witvoet J, Sedel L. Ceramic-ceramic and metal-polyethylene total hip replacements: comparison of pseudomembranes after loosening. J Bone Joint Surg Br 1997;79:135-9.

[16] Malchau H, Herberts P, Eisler T, Garellick G, Soderman P. The Swedish total hip replacement register. J Bone Joint Surg Am 2002;84A:2-20.

[17] Mandell JA, Carter DR, Goodman SB, Schurman DJ, Beaupre GS. A conical-collared intramedullary stem can improve stress transfer and limit micromotion. Clin Biomech (Bristol, Avon) 2004;19:695-703.

[18] Mjoberg B. The theory of early loosening of hip prostheses. Orthopedics 1997;20:1169-75.

[19] Nazarian A, Muller J, Zurakowski D, Muller R, Snyder BD. Densitometric, morphometric and mechanical distributions in the human proximal femur. J Biomech 2007;40:2573-9.

[20] Peter B, Gauthier O, Laib S, Bujoli B, Guicheux J, Janvier P, et al. Local delivery of bisphosphonate from coated orthopedic implants increases implants mechanical stability in osteoporotic rats. J Biomed Mater Res A 2006;76:133-43.
[21] Peter B, Pioletti DP, Laib S, Bujoli B, Pilet P, Janvier P, et al. Calcium phosphate drug delivery system: influence of local zoledronate release on bone implant osteointegration. Bone 2005;36:52-60.

[22] Pfaffl MW. A new mathematical model for relative quantification in realtime RT-PCR. Nucleic Acids Res 2001;29:e45.

[23] Pioletti DP, Muller J, Rakotomanana LR, Corbeil J, Wild E. Effect of micromechanical stimulations on osteoblasts: development of a device simulating the mechanical situation at the bone-implant interface. J Biomech 2003;36:131-5.

[24] Pioletti DP, Takei H, Kwon SY, Wood D, Sung KL. The cytotoxic effect of titanium particles phagocytosed by osteoblasts. J Biomed Mater Res 1999;46:399-407.

[25] Quinn JM, Itoh K, Udagawa N, Hausler K, Yasuda H, Shima N, et al. Transforming growth factor beta affects osteoclast differentiation via direct and indirect actions. J Bone Miner Res 2001;16:1787-94.

[26] Quinn JMW, Gillespie MT. Modulation of osteoclast formation. Biochem Biophys Res Commun 2005;328:739-45.

[27] Ramaniraka NA, Rakotomanana LR, Leyvraz PF. The fixation of the cemented femoral component. Effects of stem stiffness, cement thickness and roughness of the cement-bone surface. J Bone Joint Surg Br 2000;82 297-303.

[28] Rancourt D, Shirazi-Adl A, Drouin G, Paiement G. Friction properties of the interface between porous-surfaced metals and tibial cancellous bone. J Biomed Mater Res 1990;24:1503-19.

[29] Reckling FW, Asher MA, Dillon WL. Longitudinal-study of radiolucent line at bone-cement interface following total joint-replacement procedures. J Bone Joint Surg Am 1977;59:355-8.

[30] Simonet WS, Lacey DL, Dunstan CR, Kelley M, Chang MS, Luthy R, et al. Osteoprotegerin: a novel secreted protein involved in the regulation of bone density. Cell 1997;89:309-19.

[31] Takagi M, Santavirta S, Ida H, Ishii M, Takei I, Niissalo S, et al. Highturnover periprosthetic bone remodeling and immature bone formation around loose cemented total hip joints. J Bone Miner Res 2001;16: 79-88.

[32] Takayanagi H, Kim S, Taniguchi T. Signaling crosstalk between RANKL and interferons in osteoclast differentiation. Arthritis Res 2002;4(Suppl 3): S227-32.

[33] Terrier A, Rakotomanana RL, Ramaniraka AN, Leyvraz PF. Adaptation models of anisotropic bone. Comput Methods Biomech Biomed Engin 1997;1:47-59.

[34] Venesmaa PK, Kroger HPJ, Miettinen HJA, Jurvelin JS, Suomalainen OT, Alhava EM. Monitoring of periprosthetic BMD after uncemented total hip arthroplasty with dual-energy X-ray absorptiometry-a 3-year follow-up study. J Bone Miner Res 2001;16:1056-61.

[35] Yan T, Riggs BL, Boyle WJ, Khosla S. Regulation of osteoclastogenesis and RANK expression by TGF-beta1. J Cell Biochem 2001;83: 320-5. 